\title{
Weak deflection angle by Casimir wormhole using Gauss-bonnet theorem and its shadow
}

\author{
Wajiha Javed, ${ }^{1, *}$ Ali Hamza, ${ }^{1, \dagger}$ and Ali Övgün ${ }^{2,3, \ddagger}$ \\ ${ }^{1}$ Division of Science and Technology, University of Education, Township-Lahore, Pakistan \\ ${ }^{2}$ Instituto de Física, Pontificia Universidad Católica de Valparaíso, Casilla 4950, Valparaíso, Chile. \\ ${ }^{3}$ Physics Department, Arts and Sciences Faculty, Eastern Mediterranean University, Famagusta, North Cyprus via Mersin 10, Turkey.
}

(Dated: January 11, 2020)

\begin{abstract}
Here we calculate the deflection angle of photon by Casimir wormhole in weak limit approximation. First we calculate Gaussian optical curvature with the help of optical spacetime geometry and so we use the Gauss-Bonnet theorem on Gaussian optical metric and find deflection angle of photon by Casimir wormhole. Moreover, we calculate the photon's deflection angle in the presence of plasma medium and we also see the graphical nature of deflection angle in both cases. After calculating the deflection angle of Casimir wormhole. Now, we move towards the shadow of Casimir wormhole. After the observations of Event Horizon Telescope, the study of shadow become very important so that we plot the shapes of shadow of Casimir wormhole, and we calculate the photon geodesic around the Casimir wormhole.
\end{abstract}

PACS numbers: 95.30.Sf, 98.62.Sb, 97.60.Lf

Keywords: Relativity and gravitation; Casimir Wormhole; Gauss-Bonnet; Effect of Plasma. Shadow of Casimir Wormhole.

\section{INTRODUCTION}

Einstein and Rosen suggested the presence of traversable wormhole in 1935 which is also known as EinsteinRosen bridges [1]. A wormhole is a theoretical topological factor of the spacetime which makes alternate routes between two particular spacetimes. As of recently, nobody figure out how to demonstrate them physically, they are just scientific idea. The idea of "wormhole" was developed firstly in 1957 inside the seminal papers of Misner and Wheeler [2] and Wheeler [3] so as to give a method for having "charge without charge". Afterward, Wheeler demonstrated that wormholes would be unsteady and non-traversable for even a photon [4]. The investigation of Lorentzian wormholes in circumstance of General Relativity (GR) goes back to the basic works of Morris and Thorne in 1988 [5] where they presented a static spherical symmetric line element, they indicated that by solving the Einstein field equation, we can find the exact solutions which represent the wormhole geometries. Later on motivated by the Morris-Thorne paper, numerous scientists analyse wormhole in various viewpoints ([6]-[42]). Later on, different sorts of traversable wormholes were came up as suitable answers for the conditions of GR, admitting a various examined in a 1989 paper by Matt Visser, in this paper he popularized a way by wormhole can be built in which the navigating way doesn't go through an area of exotic matter [43]. They are called as thin-shell wormholes. Anyhow, exotic matter cause trouble for creating wormholes. As of late, it is indicated that wormholes are additionally critical to clarify the quantum entanglement [44].

Here, we calculate the deflection angle of light (DAL) from Casimir wormhole in weak field limits (WFL). In 2008, Gibbons and Werner (GW) suggested a technique to calculate the DAL by different black holes in the WFL [45]. For calculating deflection angle, first calculate the Gaussian optical metric using optical geometry and so applying the Gauss-Bonnet theorem (GBT), we can easily calculate the DAL by asymptotically flat spacetimes [45]:

$$
\alpha=-\iint_{D_{\infty}} \mathcal{K} d S
$$

that give us accurate answer for DAL. Different authors used this technique to calculate DAL by various black holes and wormholes spacetime ([46]-[55]). After that, Crisnejo and Gallo has analyzed the DAL in the presence of plasma medium [56]. Some other works can be found in Refs [57-92].

It is generally accepted that majority of the galaxies have super-massive black holes at their centers [93, 94], for example Milky Way and Messier 87 having super-massive black hole named as Sgr A. and M87. This expose the

\footnotetext{
*Electronic address: wajiha.javed@ue.edu.pk; wajihajaved84@yahoo.com

${ }^{\dagger}$ Electronic address: alihamza.ahg@gmail.com

‡Electronic address: ali.ovgun@pucv.cl; ali.ovgun@emu.edu.tr; https : / / aovgun.weebly • com/
} 
way for scientist to observe the picture of those super-massive black holes that would give solid proof with respect to their presence. Hence the investigation regarding shadow of black hole has gotten a lot of consideration recently. Due to solid gravitational impact Black Hole throws his shadow as an optical display on bright background. As a matter of fact, a viewer sees the photon circles about the event horizon. Event Horizon Telescope (EHT) is accessible experimental arrangement with respect to watch the black hole's shadow. The space experts of EHT is trying to see the picture of super-massive black hole Sgr A. with the help of BlackHoleCam. We need a earth size telescope for watching Sgr A. but it is difficult to make such telescope. That problem can be settled by the assistance of Very Long Baseline Interferometry (VLBI) organize. Moreover VLBI is a astronomical interferometry, here earth-based radio telescopes gather signals by astronomical radio stations. Those radio telescopes are circulated around the earth with the end goal is that to develope a virtual earth-sized telescope and it can deliver most highest result at the $\mathrm{mm} / \mathrm{sub}-\mathrm{mm}$ wavelength scale. Shadow of Schwarzschild black hole is examined by Synge [95] and it is additionally investigated by Luminet [96]. Bardeen [97, 98] analyzed shadow cast of Kerr black hole and more propagation was given by Falcke [99]. Some other works can be found in Refs [100-107].

Main purpose of the paper is to calculate the deflection angle in non-plasma and plasma medium and shadow caste by Casimir wormhole. For doing this paper is unionized as: In Sect. II, we calculate the Gaussian optical metric of Casimir wormhole and through GBT method by GW, we calculate its deflection angle in Sect. III. In Sect. IV, we observe the graphical behavior of deflection angle in non-plasma. In Sect. V, we calculate the DAL by Casimir wormhole in plasma medium. In Sect. VI, we analyzed the plots of Casimir wormhole in plasma medium, next we find the null geodesic in Sect. VII and in Sect. VIII we find the shadow of Casimir wormhole and finally in Sect. IX, we conclude our result.

\section{OPTICAL METRIC OF CASIMIR WORMHOLE}

The metric of a Casimir traversable wormhole in a spherically symmetric spacetime is define as [108];

$$
d s^{2}=-A(r) d t^{2}+\frac{d r^{2}}{B(r)}+r^{2} d \Omega^{2}
$$

Where $A(r)$ and $B(r)$ is defined as

$$
A(r)=\left(\frac{3 r}{3 r+a}\right)^{2}, B(r)=1-\frac{2 a}{3 r}-\frac{a^{2}}{3 r^{2}} \text { and } d \Omega^{2}=d \theta^{2}+\sin ^{2} \theta d \phi^{2} .
$$

Here $a$ is a constant and $r$ is a radial coordinate such that $r \in[a, \infty]$. After expanding $A(r)$ as a series and take only first order of $a$, we can write $\mathrm{A}(\mathrm{r})$ as :

$$
A(r)=1-\frac{2 a}{3 r} .
$$

As cause and viewer are in the tropical plane and null photon is also in a similar plane, so we can assume $\left(\theta=\frac{\pi}{2}\right)$. For getting optical metric we put $d s^{2}=0$,

$$
d t^{2}=\frac{d r^{2}}{A(r) B(r)}+\frac{r^{2} d \phi^{2}}{A(r)}
$$

Now by using Eq. (3), the non-zero christofell symbols are defined as

$$
\Gamma_{00}^{0}=-\frac{B^{\prime}(r)}{2 B(r)}-\frac{A^{\prime}(r)}{2 A(r)} \quad, \quad \Gamma_{01}^{1}=\frac{1}{r}-\frac{A^{\prime}(r)}{2 A(r)}
$$

and

$$
\Gamma_{11}^{0}=-r B(r)+\frac{r^{2} A^{\prime}(r) B(r)}{2 A(r)},
$$

and RicciScalar related to the optical metric is calculated as:

$$
\begin{aligned}
\mathcal{R} & =-\frac{A(r) B^{\prime}(r)}{r}+\frac{A^{\prime}(r) B^{\prime}(r)}{2}+\frac{A^{\prime}(r) B(r)}{r}+A^{\prime \prime}(r) B(r) \\
& -\frac{\left(A^{\prime}(r)\right)^{2} B(r)}{2 A(r)} .
\end{aligned}
$$


The Gaussian optical curvature that is calculated as follows:

$$
\mathcal{K}=\frac{\text { RicciScalar }}{2}
$$

After simplifying, Gaussian optical curvature is given as:

$$
\mathcal{K} \approx \frac{-3 a+2 a^{2}}{9}\left(\frac{1}{r^{3}}\right)+\frac{-15 a^{2}+4 a^{3}}{27}\left(\frac{1}{r^{4}}\right)+\mathcal{O}\left(\frac{1}{r^{5}}\right) .
$$

\section{DEFLECTION ANGLE OF CASIMIR WORMHOLE}

Now by using the GBT we can obtain the DAL of Casimir wormhole. We use the GBT to the area $\mathcal{E}_{S}$, given as [45]

$$
\iint_{\mathcal{E}_{S}} \mathcal{K} d S+\oint_{\partial \mathcal{E}_{S}} k d t+\sum_{j} \epsilon_{j}=2 \pi \mathcal{Y}\left(\mathcal{E}_{S}\right)
$$

and Gaussian curvature is indicated by $\mathcal{K}$ and geodesic curvature is indicated by $k$, declared as $k=\bar{g}\left(\nabla_{\dot{\alpha}} \dot{\alpha}, \ddot{\alpha}\right)$ in this way as $\bar{g}(\dot{\alpha}, \dot{\alpha})=1, \ddot{\alpha}$ represents unit acceleration vector and the $\epsilon_{j}$ represents the exterior angle at the jth vertex. As $S \rightarrow \infty$, both the jump angles reduce to $\pi / 2$ and we get $\theta_{O}+\theta_{S} \rightarrow \pi$. The Euler characteristic is $\mathcal{Y}\left(\mathcal{E}_{S}\right)=1$, as $\mathcal{E}_{S}$ is non singular. So,

$$
\iint_{\mathcal{E}_{S}} \mathcal{K} d S+\oint_{\partial \mathcal{E}_{S}} k d t+\epsilon_{j}=2 \pi \mathcal{Y}\left(\mathcal{E}_{S}\right)
$$

here, $\epsilon_{j}=\pi$ shows that both $\alpha_{\bar{g}}$ and the total jump angle is a geodesic, since the Euler characteristic number expressed by $\mathcal{Y}$ is 1 . As $S \rightarrow \infty$, the only interesting part to be determined is $k\left(D_{S}\right)=\left|\nabla_{\dot{D}_{S}} \dot{D}_{S}\right|$. Since, radial component of geodesic curvature is defined as [45]

$$
\left(\nabla_{\dot{D}_{S}} \dot{D}_{S}\right)^{r}=\dot{D}_{S}^{\phi} \partial_{\phi} \dot{D}_{S}^{r}+\Gamma_{11}^{0}\left(\dot{D}_{S}^{\phi}\right)^{2}
$$

For large $S, D_{S}:=r(\phi)=S=$ const. Hence, the form of the Eq. (8) becomes $\left(\dot{D}_{S}^{\phi}\right)^{2}=\frac{A^{2}(r) B(r)}{r^{2}}$. As $\Gamma_{11}^{0}=$ $-r B(r)+\frac{r^{2} A^{\prime}(r) B(r)}{2 A(r)}$, so it becomes

$$
\left(\nabla_{\dot{D}_{S}^{r}} \dot{D}_{S}^{r}\right)^{r} \rightarrow \frac{-1}{S}
$$

As topological defect is not present in geodesic curvature so, $k\left(D_{S}\right) \rightarrow S^{-1}$. But with the help of optical metric Eq. (5), we can write as $d t=S d \phi$. Hence, we get this;

$$
k\left(D_{S}\right) d t=\frac{1}{S} S d \phi
$$

With the help of all above results, we obtained

$$
\iint_{\mathcal{E}_{S}} \mathcal{K} d s+\oint_{\partial \mathcal{E}_{S}} k d t={ }^{S \rightarrow \infty} \iint_{T_{\infty}} \mathcal{K} d S+\int_{0}^{\pi+\Theta} d \phi
$$

Light ray at 0 th order in week field limit is defined as $r(t)=b / \sin \phi$. Now, using (6) and (12), the DAL defined as [45]

$$
\Theta=-\int_{0}^{\pi} \int_{b / \sin \phi}^{\infty} \mathcal{K} \sqrt{\operatorname{det} \bar{g}} d r d \phi
$$

where

$$
\sqrt{\operatorname{det} \bar{g}}=\left(\frac{2 a}{3}+\frac{2 a^{2}}{9}\right)+r+\frac{r a}{3}+\frac{a^{2}}{6 r}+\frac{a^{3}}{18 r^{2}} d r
$$

After putting the leading order terms of Gaussian curvature Eq. (5) into Eq. (12), the deflection angle is defined as:

$$
\Theta \approx \frac{-4}{b}\left[\frac{-a}{3}+\frac{a^{2}}{9}+\frac{2 a^{3}}{27}\right]+\mathcal{O}\left(\frac{1}{b^{2}}\right)
$$




\section{GRAPHICAL ANALYSIS FOR NON-PLASMA MEDIUM}

Here, in this portion we talk about the graphical behavior of DAL. We observe the relation of deflection angle $\Theta$ with impact parameter $b$ with the variation of $a$.

\section{A. Deflection angle $\Theta$ w.r.t Impact parameter $b$}
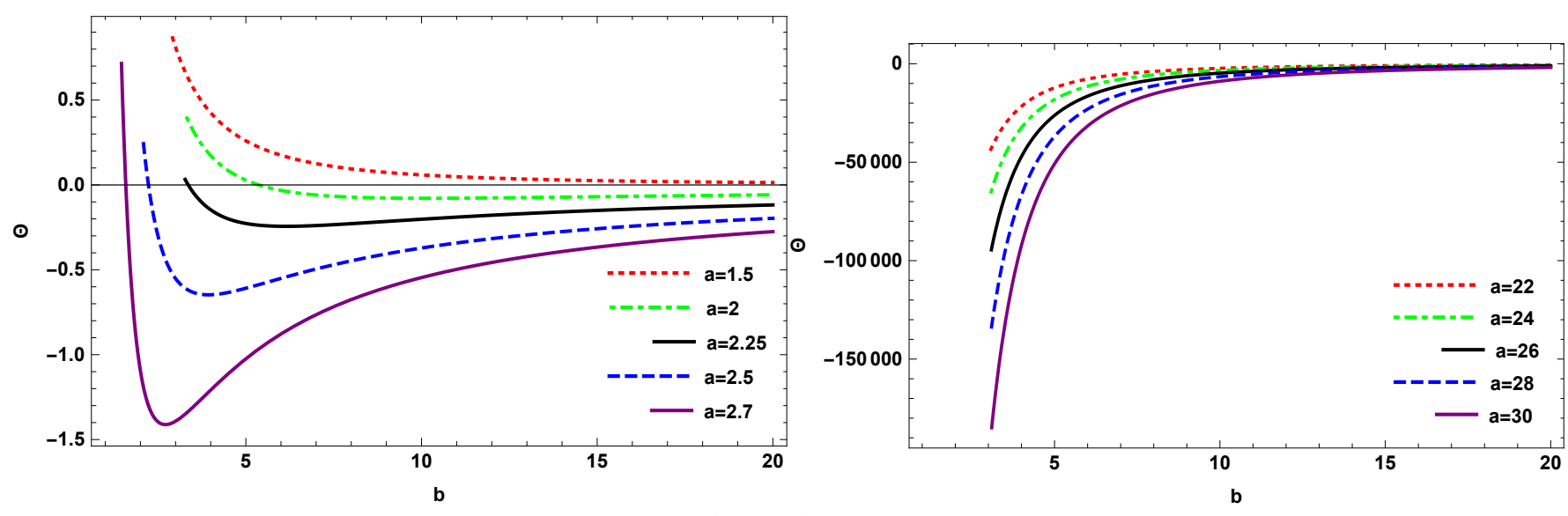

Figure 1: Relation between $\Theta$ and $b$.

- Figure 1 display the relation of $\Theta$ w.r.t $b$ for different values of $a$.

1. In left plot, we saw that for small values of $a$ the deflection angle first decreasing from positive to negative and then gradually increasing that indicates the balanced behavior.

2. In right figure, we observed that for choosing large values of $a$ deflection angle is continuously decreasing from positive to negative.

\section{EFFECT OF PLASMA ON GRAVITATIONAL LENSING}

This segment is based on the investigation of DAL by Casimir wormhole in the presence of plasma medium. For Casimir wormhole the refractive index $n(r)$ [56], is obtain as,

$$
n^{2}[r, \omega(r)]=1-\frac{\omega_{e}^{2}(r)}{\omega_{\infty}^{2}(r)} .
$$

Refractive index is defined as;

$$
n(r)=\sqrt{1-\frac{\omega_{e}^{2}}{\omega_{\infty}^{2}}(A(r))} .
$$

Where $\omega_{e}$ is electron plasma frequency and $\omega_{\infty}$ is photon frequency evaluated by an viewer at infinity and so the corresponding optical metric defined as [56]

$$
d t^{2}=g_{l m}^{o p t} d x^{l} d x^{m}=n^{2}\left[\frac{d r^{2}}{A(r) B(r)}+\frac{r^{2} d \phi^{2}}{A(r)}\right] .
$$

Here the metric function of the above optical metric is given as

$$
A(r)=1-\frac{2 a}{3 r} \text { and } B(r)=1-\frac{2 a}{3 r}-\frac{a^{2}}{3 r^{2}},
$$

the non-zero christopher symbols corresponding of optical metric are defined as:

$$
\Gamma_{00}^{0}=\left(1+\frac{\omega_{e}^{2} A(r)}{\omega_{\infty}^{2}}\right)\left[-\frac{A^{\prime}(r) B(r)+A(r) B^{\prime}(r)}{2 A(r) B(r)}\left(1-\frac{\omega_{e}^{2} A(r)}{\omega_{\infty}^{2}}\right)-\frac{\omega_{e}^{2} A^{\prime}(r)}{2 \omega_{\infty}^{2}}\right],
$$




$$
\Gamma_{10}^{1}=\left(1+\frac{\omega_{e}^{2} A(r)}{\omega_{\infty}^{2}}\right)\left[\frac{1}{r}\left(1-\frac{\omega_{e}^{2} A(r)}{\omega_{\infty}^{2}}\right)-\frac{A^{\prime}(r)}{2 A(r)}\left(1-\frac{\omega_{e}^{2} A(r)}{\omega_{\infty}^{2}}\right)-\frac{\omega_{e}^{2} A(r)}{2 \omega_{\infty}^{2}}\right]
$$

and

$$
\begin{aligned}
\Gamma_{11}^{0} & =\left(1+\frac{\omega_{e}^{2} A(r)}{\omega_{\infty}^{2}}\right)\left[-r B(r)\left(1-\frac{\omega_{e}^{2} A(r)}{\omega_{\infty}^{2}}\right)+\frac{r^{2} B(r)}{2} \frac{\omega_{e}^{2} A^{\prime}(r)}{\omega_{\infty}^{2}}\right. \\
& \left.+\frac{r^{2} A^{\prime}(r) B(r)}{2 A(r)}\left(1-\frac{\omega_{e}^{2} A(r)}{\omega_{\infty}^{2}}\right)\right] .
\end{aligned}
$$

Gaussian curvature in terms of curvature tensor can be determined as

$$
\mathcal{K}=\frac{R_{r \phi r \phi}\left(g^{o p t}\right)}{\operatorname{det}\left(g^{o p t}\right)}
$$

with the help of Eq. (20) Gaussian curvature is written as

$$
\begin{aligned}
\mathcal{K} & \approx\left[\frac{-3 a+2 a^{2}}{9}+\frac{\omega_{e}^{2}}{\omega_{\infty}^{2}}\left(\frac{-15 a+32 a^{2}}{36}\right)+\frac{\omega_{e}^{4}}{\omega_{\infty}^{4}}\left(\frac{9 a-6 a^{2}}{36}\right)\right]\left(\frac{1}{r^{3}}\right) \\
& +\left[\frac{-15 a^{2}+4 a^{3}}{27}+\frac{\omega_{e}^{2}}{\omega_{\infty}^{2}}\left(\frac{-27 a^{2}+10 a^{3}}{36}\right)+\frac{\omega_{e}^{4}}{\omega_{\infty}^{4}}\left(\frac{-21 a^{2}+14 a^{3}}{36}\right)\right]\left(\frac{1}{r^{4}}\right) \\
& +\mathcal{O}\left(\frac{1}{r^{5}}\right) .
\end{aligned}
$$

By using GBT, we check the DAL in order to compare it with non-plasma. In this way, for finding angle in the WFL, as the light beams pursues a straight line estimation so we utilize a case of $r=\frac{b}{\sin \phi}$ at 0th order.

$$
\Theta=-\lim _{R \rightarrow 0} \int_{0}^{\pi} \int_{\frac{b}{\sin \phi}}^{R} \mathcal{K} d S .
$$

Where $d S=\sqrt{g} d r d \phi$ and

$$
\sqrt{g}=\frac{6 a+2 a^{2}}{9}+\left[\frac{3+a}{3}\left(1-\frac{\omega_{e}^{2}}{\omega_{\infty}^{2}}\right)\right] r+\left[\frac{a^{2}}{6}\left(1-\frac{\omega_{e}^{2}}{\omega_{\infty}^{2}}\right)\right] \frac{1}{r}+\mathcal{O}\left(\frac{1}{r^{2}}\right)
$$

With the help of Eq. (11), the DAL in plasma medium is defined as;

$$
\begin{aligned}
\Theta & \approx \frac{-4}{b}\left[\frac{-a}{3}+\frac{a^{2}}{9}+\frac{2 a^{3}}{27}-\frac{a \omega_{e}^{2}}{12 \omega_{\infty}^{2}}+\frac{23 a^{2} \omega_{e}^{2}}{36 \omega_{\infty}^{2}}+\frac{2 a^{3} \omega_{e}^{2}}{9 \omega_{\infty}^{2}}+\frac{2 a \omega_{e}^{4}}{3 \omega_{\infty}^{4}}-\frac{5 a^{2} \omega_{e}^{4}}{6 \omega_{\infty}^{4}}\right. \\
& \left.-\frac{19 a^{3} \omega_{e}^{4}}{54 \omega_{\infty}^{4}}\right]+\mathcal{O}\left(\frac{\omega_{e}^{6}}{\omega_{\infty}^{6}}, \frac{1}{b^{2}}\right) .
\end{aligned}
$$

The above results tells us that photon rays are moving into medium of homogeneous plasma. We see that Eq. (23) reduced into Eq. (14) if plasma effect can be removed.

\section{GRAPHICAL ANALYSIS FOR PLASMA MEDIUM}

Now, we want to observe the deflection angle of Casimir wormhole in the presence of plasma medium. Here, we take $\frac{\omega_{e}}{\omega_{\infty}}=10^{-1}$ and observe behavior of DAL by changing different values of $a$. 


\section{A. Deflection angle w.r.t Impact parameter}
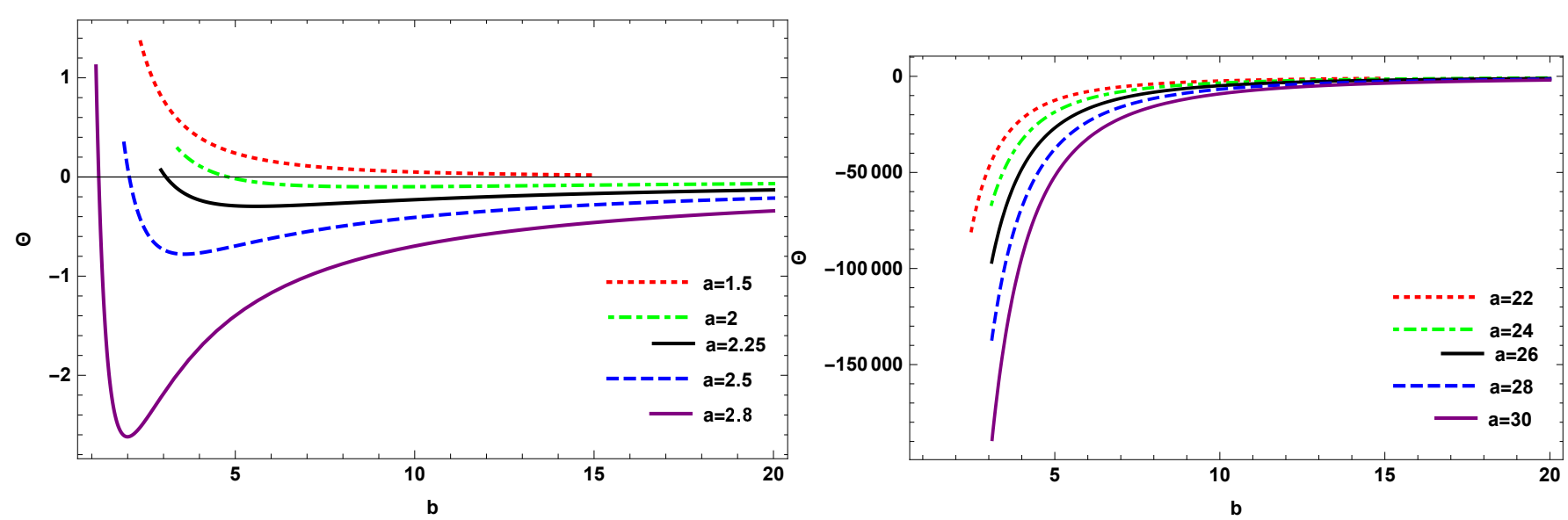

Figure 2: Relation between $\Theta$ and $b$.

- Figure 2 demonstrates the relation of $\Theta$ with $b$ for different values of $a$.

1. In 1st plot we examined that for small values of $a$ the DAL first decreasing from positive to negative and then gradually increasing as like in Figure 1.

2. In 2nd plot we observed that DAL gradually decrease for large values of $a$ as like in Figure $\mathbf{1 .}$

\section{NULL GEODESIC IN A CASIMIR WORMHOLE}

The Lagrangian identifying the motion of a photon in the spacetime of the Casimir wormhole Eq. (2) is given by

$$
2 \mathcal{L}=-\left(1-\frac{2 a}{3 r}\right) \dot{t}^{2}+\left(\frac{1}{1-\frac{2 a}{3 r}-\frac{a^{2}}{3 r^{2}}}\right) \dot{r}^{2}+r^{2} \dot{\theta}^{2}+r^{2} \sin ^{2} \theta \dot{\phi}^{2},
$$

where an overdot means derivative w.r.t the affine parameter $\lambda$. As the Lagrangian is independent of $\phi$ and $t$ so there are only two constants of motion named as the energy $E$ and the angular momentum $L$. Where $E$ and $L$ are defined as:

$$
p_{t}=\frac{\partial L}{\partial \dot{t}}=-\left(1-\frac{2 a}{3 r}\right) \dot{t}=-E
$$

and

$$
p_{\phi}=\frac{\partial L}{\partial \dot{\phi}}=r^{2} \sin ^{2} \theta \dot{\phi}=L
$$

Now we can determine the geodesic conditions by utilizing these preserved measures

$$
\frac{d t}{d \lambda}=\dot{t}=\frac{E}{1-\frac{2 a}{3 r}} \quad, \quad \frac{d \phi}{d \lambda}=\dot{\phi}=\frac{L}{r^{2} \sin ^{2} \theta} .
$$

The $r$-part and $\theta$-part of the momentum are defined as

$$
p_{r}=\frac{\partial L}{\partial \dot{r}}=\frac{\dot{r}}{1-\frac{2 a}{3}-\frac{a^{2}}{3 r^{2}}} \text { and } p_{\phi}=\frac{\partial L}{\partial \dot{\phi}}=r^{2} \dot{\theta} .
$$

The $r$-part and $\theta$-part of the geodesic equations can be calculated by working on the Hamilton-Jacobi equation

$$
\frac{\partial S}{\partial \lambda}=-\frac{1}{2} g^{\mu \nu} \frac{\partial S}{\partial x^{\mu}} \frac{\partial S}{\partial x^{\nu}},
$$


and for photons $\left(m_{0}=0\right)$, Eq. (27) has a result of the succeeding type

$$
S=-E t+L \phi+S_{r}(r)+S_{\theta}(\theta),
$$

here $S_{r}$ is a function or $r$ and $S_{\theta}$ is the function of $\theta$. By putting Eq. (28) into Eq. (27) and also putting the value of contravariant metric, i.e., $g^{\mu \nu}$, and differentiate the terms of variables $r$ and $\theta$ equal to the Carter constant $( \pm \mathcal{K})$ [109], we get

$$
\begin{aligned}
\frac{1}{\sqrt{1-\frac{2 a}{3}-\frac{a^{2}}{3 r^{2}}}} \frac{d r}{d \lambda} & ={ }_{-}^{+} \sqrt{R}(r), \\
r^{2} \frac{d \theta}{d \lambda} & ={ }_{-}^{+} \sqrt{T}(\theta),
\end{aligned}
$$

here $R$ and $\theta$ can be written as

$$
\begin{array}{r}
R(r)=\frac{E^{2}}{1-\frac{2 a}{3 r}}-\frac{\mathcal{K}}{r^{2}}, \\
T(\theta)=\mathcal{K}-\frac{L^{2}}{\sin ^{2} \theta} .
\end{array}
$$

As we have geodesic equations now our main concern is to find the radial motion of photons across the throat of Casimir wormhole, that can be showed by the computation of the effective potential

$$
\frac{d r^{2}}{d \lambda}+V_{e f f}=0
$$

with

$$
V_{\text {eff }}=-\left(1-\frac{2 a}{3}-\frac{a^{2}}{3 r^{2}}\right) R(r)
$$

From the expression we can say that effective potential depend on constant $a$, radial coordinate $r$ and on energy $E$. It is advantageous to decrease the quantity of parameters by characterizing the impact parameters such as $\xi=\frac{L}{E}$ and $\eta=\frac{\mathcal{K}}{E^{2}}$. Photon geodesics can be communicated as far as these impact parameters $(\xi, \eta)$. Now we write $\mathcal{R}$, in the form of impact parameters as pursues

$$
(R)=E^{2}\left[\frac{1}{1-\frac{2 a}{3 r}}-\frac{\eta}{r^{2}}\right] .
$$

The investigation of effective potential expose the presence of un-stable circular orbits of fixed radius about the throat of wormholes. Those circles are significant from the perspective of optical perceptions.

\section{SHADOW OF CASIMIR WORMHOLE}

Here, we explore the shadow cast of Casimir wormhole. As we talk about the wormhole that it is a tunnel-like shape relating various areas of space-time and it doesn't have any horizon or singularity inside it. Also, it is thought that gravitational force of a wormhole is so weak that a human can tolerate it easily. Wormhole's throat is very important because unstable photon present here and they are spinning about throat infinite times before reaching to the viewer.

Let us assume the circumstance here one of the space-time areas that is associated with wormholes is lighted with a light source and a far eyewitness is additionally present here. One would note this, in the other space-time area there is no light source present and hence photons cannot navigate from this area. As an far eyewitness can have the option to watch the disorder photons. Moreover, the photons that are plunging, subsequently navigating from the wormholes and can't be seen by the eyewitness. The nonattendance of the navigated photons forms a dark spot on the bright background known as shadow of wormhole. The non-stable round photons give the boundary of the shadow. Conditions that check the non-stable round photons can be written in this form

$$
R=0, \quad R^{\prime}=0 .
$$


Here prime ( $I$ ) denote derivative w.r.t $r$. Putting (33) into (34), we can easily get

$$
\eta=\frac{r^{2}}{1-\frac{2 a}{3 r}}, \quad r=\frac{4 a}{3} .
$$

Here, impact parameter $\eta$ depend on radial coordinate $r$ and on constant $a$. Eq. (35) defines the shadow's boundary and a far eyewitness can note its projection in his sky. Therefor, we make the celestial coordinates $(\alpha, \beta)$ in the eyewitness's sky and associate them with impact parameters $(\xi, \eta)$. The celestial coordinates written ([110],[111]) as

$$
\begin{array}{r}
\alpha=\lim _{r_{0} \rightarrow \infty}\left(r_{0}^{2} \sin \theta_{0}\right) \frac{d \phi}{d r}, \\
\beta=\lim _{r_{0} \rightarrow \infty} r_{0}^{2} \frac{d \theta}{d r},
\end{array}
$$

here distance between wormhole and observer is denoted by $r_{0}$ and $\theta_{0}$ is observer's angular coordinate or we can say it "inclination angle". Now putting the equations of four-velocities into Eq. (36), and doing some simple direct calculations we get this type of celestial coordinates

$$
\alpha=-\frac{\xi}{\sin \theta_{0}} \quad \text { and } \quad \beta=\sqrt{\eta-\frac{\xi^{2}}{\sin \theta_{0}^{2}}} .
$$

Knowing the equations of celestial coordinates and impact parameters, we now make the shadow of Casimir wormhole. So as to construct the shape of shadow, we plot $\alpha$ versus $\beta$ which gives the shadow's boundary in the eyewitness's sky. The plots of the shadow for the Casimir wormholes can be saw from Fig 3.
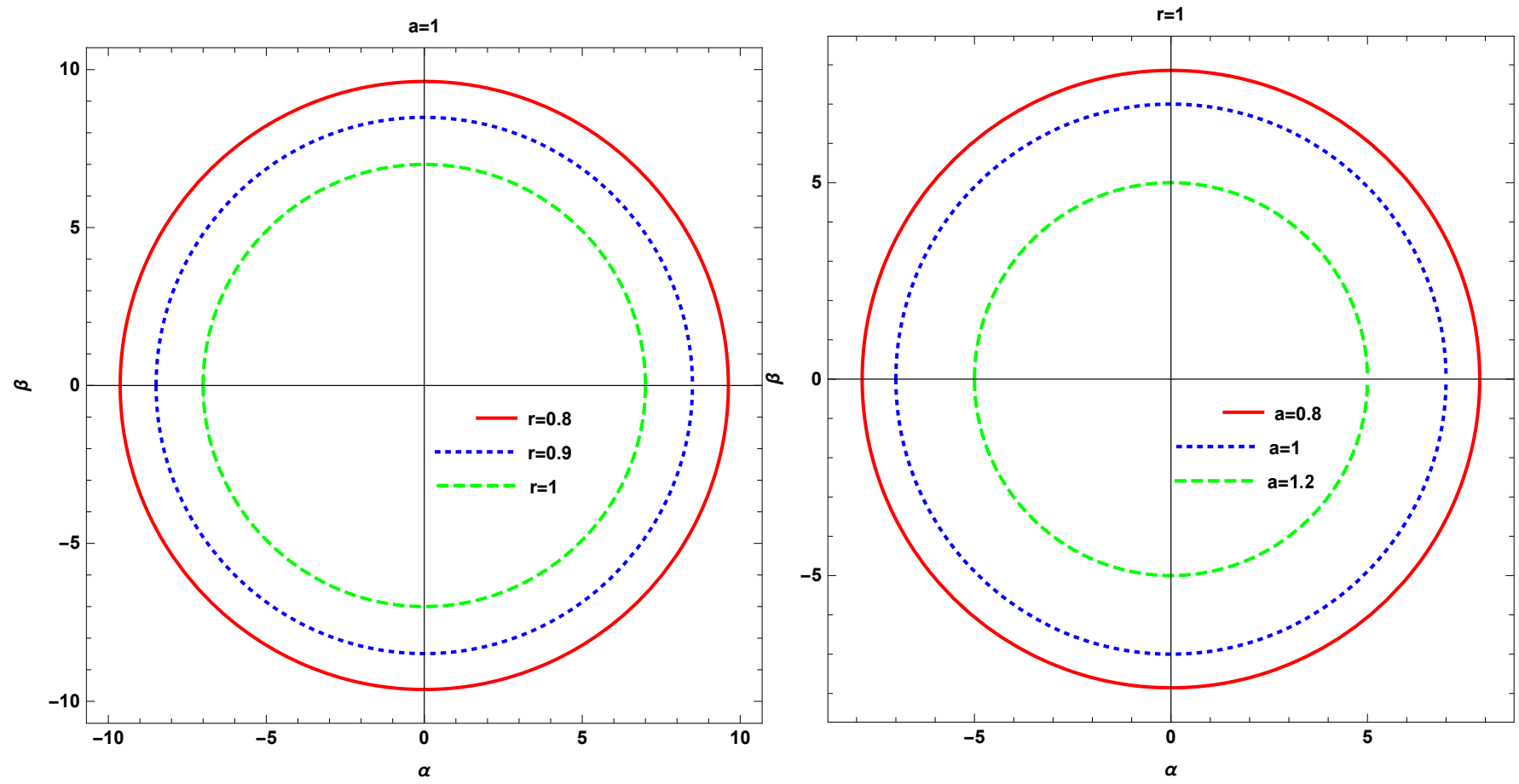

Figure 3: Graphs displaying the shadow of Casimir wormhole.

By changing the values of constant $(a)$ and radial coordinate $(r)$, we check the behavior of shadow of Casimir wormhole in the equatorial plan by putting $\theta_{0}=\frac{\pi}{2}$. We can see from the plots that shadow's shape is a perfect square and it is changed by changing values of $(a)$ and $(r)$ as shown in Fig. 3. It is seen from the graphs that shadow of Casimir wormhole reduces by increasing the values of $(a)$ and $(r)$.

\section{CONCLUSION}

In this paper, first of all we use the GBT to find the DAL by Casimir wormhole. For this we obtain the optical gaussian curvature with the help of optical metric and apply the GBT on optical gaussian curvature and calculated 
the DAL. The deflection angle is defined as

$$
\Theta \approx \frac{-4}{b}\left[\frac{-a}{3}+\frac{a^{2}}{9}+\frac{2 a^{3}}{27}\right]
$$

This shows that deflection angle only depends on the impact parameter. Next we also check the graphical behavior of this deflection angle. In this behavior we analyzed that for small values of $(a)$ deflection angle first decreasing and then goes to positive increase and for large values of $(a)$ deflection angle continue decreasing from positive to negative side. We also discuss the deflection angle of Casimir wormhole in the presence of plasma medium. Deflection angle of Casimir wormhole in the presence of plasma medium is defined as

$$
\begin{aligned}
\Theta & \approx \frac{-4}{b}\left[\frac{-a}{3}+\frac{a^{2}}{9}+\frac{2 a^{3}}{27}-\frac{a \omega_{e}^{2}}{12 \omega_{\infty}^{2}}+\frac{23 a^{2} \omega_{e}^{2}}{36 \omega_{\infty}^{2}}+\frac{2 a^{3} \omega_{e}^{2}}{9 \omega_{\infty}^{2}}+\frac{2 a \omega_{e}^{4}}{3 \omega_{\infty}^{4}}-\frac{5 a^{2} \omega_{e}^{4}}{6 \omega_{\infty}^{4}}\right. \\
& \left.-\frac{19 a^{3} \omega_{e}^{4}}{54 \omega_{\infty}^{4}}\right] .
\end{aligned}
$$

Here we note that this deflection angle is also depend only on the impact parameter. Now if we neglect the plasma effect $\left(\frac{\omega_{e}}{\omega_{\infty}} \rightarrow 0\right)$ then this deflection angle Eq. (23) reduces into this angle Eq. (14). We also observed the graphical behavior in the presence of plasma medium. In these plots we observed that these plots shows the same behavior as in the non-plasma plots.

Now in the 2nd portion we calculate the shadow of Casimir wormhole. Since most recent couple of decades the investigation of shadow cast by the compact articles particularly for black hole and wormholes has been talked about conspicuously. Since subject has astrophysical importance in light of the fact that solid confirmations recommend the presence of supermassive black hole at the center of giant galaxies. The examination is significant from the perspective of talking about the idea of these astrophysical items. According to prediction of general relativity wormholes behave as tunnel like passage, as a two dirction interface, a connection between two space-times that are in same Universe or not. So here, in this portion, we first of all calculate the Null Geodesic of Casimir wormhole and then we make the shadow of Casimir wormhole. We have calculated the celestial co-ordinates and impact parameters to see the shape of Casimir wormhole's shadow. We saw that its shadow is a perfect circle and this circle change by changing values of $r$ and $a$ and we make the plots for different cases. Result of these graph is that radius of this shadow decrease when we increase the value of $r$ and $a$.

\section{Acknowledgments}

This work was supported by Comisión Nacional de Ciencias y Tecnología of Chile through FONDECYT Grant $N^{\circ}$ 3170035 (A. Ö.).

[1] A. Einstein and N. Rosen, Phys. Rev. 48, 73-77 (1935).

[2] C. W. Misner and J. A. Wheeler, Ann. Phys. 2, 525 (1957); C. W. Misner, Phys. Rev. 118, 1110 (1959).

[3] J. A. Wheeler, Ann. Phys. 2, 604 (1957); J. A. Wheeler, Geometrodynamics (Academic, New York, 1962).

[4] J. A. Wheeler, Phys. Rev. 97, 511 (1955); R. W. Fuller and J. A. Wheeler, Phys. Rev. 128, 919 (1962).

[5] M. S. Morris and K. S. Thorne, Am. J. Phys. 56, 395 (1988); M. S. Morris, K. S. Thorne and U. Yurtsever, Phys. Rev. Lett. 61, $1446(1988)$.

[6] T. Damour and S. N. Solodukhin, Phys. Rev., D76:024016, 2007.

[7] P. Bueno, P. A. Cano, F. Goelen, T. Hertog, and B. Vercnocke, Phys. Rev., D97(2):024040, 2018.

[8] M. Visser, Phys. Rev., D39:3182-3184, 1989.

[9] F. S. N. Lobo, Phys. Rev., D71:124022, 2005.

[10] J. P. S. Lemos, F. S. N. Lobo, and S. Q. de Oliveira, Phys. Rev., D68:064004, 2003.

[11] J. Maldacena and L. Susskind, Fortsch. Phys., 61:781-811, 2013.

[12] S. W. Hawking, Phys. Rev., D37:904-910, 1988.

[13] S. V. Sushkov, Phys. Rev., D71:043520, 2005.

[14] K. A. Bronnikov and S.-W. Kim, Phys. Rev., D67:064027, 2003.

[15] V. P. Frolov and I. D. Novikov, Phys. Rev., D42:1057-1065, 1990.

[16] M. S. R. Delgaty and R. B. Mann, Int. J. Mod. Phys., D4:231-246, 1995.

[17] G. P. Perry and Robert B. Mann, Gen. Rel. Grav., 24:305-321, 1992.

[18] J. G. Cramer, R. L. Forward, M. S. Morris, M. Visser, G. Benford, and G. A. Landis, Phys. Rev., D51:3117-3120, 1995. 
[19] J. Oliva, D. Tempo, and R. Troncoso, JHEP, 07:011, 2009.

[20] J. M. Maldacena and L. Maoz, JHEP, 02:053, 2004.

[21] G. Clement, Gen. Rel. Grav., 16:131, 1984.

[22] G. Clement, Phys. Rev., D51:6803-6809, 1995.

[23] G. Clement, J. Math. Phys., 38:5807-5819, 1997.

[24] E. I. Guendelman, Gen. Rel. Grav., 23:1415-1419, 1991.

[25] E. I Guendelman, A. Kaganovich, E. Nissimov, and S. Pacheva, Phys. Lett., B681:457-462, 2009.

[26] E. I. Guendelman, A. Kaganovich, E. Nissimov, and S. Pacheva, Int. J. Mod. Phys., A25:1405-1428, 2010.

[27] E. Guendelman, A. Kaganovich, E. Nissimov, and S.Pacheva, Phys. Lett., B673:288-292, 2009.

[28] I. Sakalli and A. Ovgun, Eur. Phys. J. Plus, 130(6):110, 2015.

[29] I. Sakalli and A. Ovgun, Astrophys. Space Sci., 359(1):32, 2015.

[30] M. G. Richarte, I. G. Salako, J. P. Morais Graca, H. Moradpour, and A. Övgün, Phys. Rev., D96(8):084022,2017.

[31] A. Övgün, K. Jusufi, and I. Sakalli, Phys. Rev. D 99, no. 2, 024042 (2019).

[32] A. Övgün, arXiv:1803.04256.

[33] A. Övgün and K. Jusufi, Eur. Phys. J. Plus, 132(12):543, 2017.

[34] A. Övgün, Eur. Phys. J. Plus, 131(11):389, 2016.

[35] A. Övgün and M. Halilsoy, Astrophys.Space Sci., 361(7):214, 2016.

[36] K.Jusufi, A. Övgün, A. Banerjee, and I. Sakalli, Eur. Phys. J. Plus 134, no. 9, 428 (2019).

[37] K. Jusufi, A. Övgün, and A. Banerjee, Phys. Rev., D96(8):084036, 2017.

[38] K. Jusufi and A. Övgün, Phys. Rev., D97(2):024042, 2018.

[39] M. Halilsoy, A. Ovgun, and S. Habib Mazharimousavi, Eur. Phys. J., C74:2796, 2014.

[40] M. Azreg-Ainou and G. Clement, Gen. Rel. Grav., 25:881-891, 1993.

[41] G. W. Gibbons and M. S. Volkov, Phys. Rev., D96(2):024053, 2017.

[42] W. T. Kim, J. J. Oh, and M. S. Yoon, Phys. Rev., D70:044006, 2004.

[43] M. Visser, Lorentzian Wormholes: From Einstein to Hawking (American Institute of Physics, New York, 1995).

[44] Donald Marolf and Joseph Polchinski, Phys.Rev.Lett. 111, 171301 (2013) .

[45] G.W. Gibbons and M. C.Werner, Class. Quant. Grav. 25, 235009(2008).

[46] G.W. Gibbons and C. M.Warnick, Phys. Rev. D 79, 064031 (2009).

[47] G.W. Gibbons, C. A. R. Herdeiro, C. M.Warnick and M. C.Werner, Phys. Rev. D 79, 044022 (2009).

[48] G.W. Gibbons and M. Vyska, Class. Quant. Grav. 29, 065016 (2012).

[49] C. Bloomer, arXiv:1111.4998 [math-ph].

[50] M. C.Werner, Gen. Rel. Grav. 44, 3047 (2012).

[51] G.W. Gibbons, Class. Quant. Grav. 33, no. 2, 025004 (2016).

[52] A. Ishihara, Y. Suzuki, T. Ono, T. Kitamura and H. Asada, Phys. Rev. D 94, no. 8, 084015 (2016).

[53] P. Das, R. Sk and S. Ghosh, Eur. Phys. J. C 77, no. 11, 735 (2017).

[54] I. Sakalli and A. Ovgun, EPL 118, no. 6, 60006 (2017).

[55] K. Jusufi, M. C.Werner, A. Banerjee and A. Övgün, Phys. Rev. D 95, no. 10, 104012 (2017).

[56] G. Crisnejo, E. Gallo, Phys. Rev. D 97(12), 124016 (2018).

[57] T. Ono, A. Ishihara and H. Asada, Phys. Rev. D 96, no. 10, 104037 (2017).

[58] K. Jusufi, I. Sakalli and A. Övgün, Phys. Rev. D 96, no. 2, 024040 (2017).

[59] A. Ishihara, Y. Suzuki, T. Ono and H. Asada, Phys. Rev. D 95, no. 4, 044017 (2017).

[60] H. Arakida, Gen. Rel. Grav. 50, no. 5, 48 (2018).

[61] A. Övgün, I. Sakalli and J. Saavedra, JCAP 1810, no. 10, 041 (2018)

[62] A. Övgün, I. Sakalli and J. Saavedra, Annals Phys. 411, 167978 (2019).

[63] A. Övgün, G. Gyulchev and K. Jusufi, Annals Phys. 406, 152 (2019).

[64] K. Jusufi, A. Övgün, J. Saavedra, P. A. Gonzalez and Y. Vasquez, Phys. Rev. D 97, 124024 (2018).

[65] A. Övgün, K. Jusufi and I. Sakalli, Annals Phys. 399, 193 (2018).

[66] K. Jusufi and A. Övgün, Phys. Rev. D 97, no. 6, 064030 (2018).

[67] A. Övgün, Phys. Rev. D 98, no. 4, 044033 (2018)

[68] T. Ono, A. Ishihara and H. Asada, Phys. Rev. D 98, no. 4, 044047 (2018)

[69] T. Ono, A. Ishihara and H. Asada, Phys. Rev. D 99, no. 12, 124030 (2019).

[70] G. Crisnejo, E. Gallo and A. Rogers, Phys. Rev. D 99, no. 12, 124001 (2019).

[71] Z. Li and A. Övgün, doi:10.20944/preprints201911.0195.v1

[72] Z. Li and T. Zhou, arXiv:1908.05592 [gr-qc].

[73] Z. Li and J. Jia, arXiv:1912.05194 [gr-qc].

[74] Z. Li, G. He and T. Zhou, arXiv:1908.01647 [gr-qc].

[75] A. Övgün, Universe 5, no. 5, 115 (2019).

[76] W. Javed, R. Babar, A. Ovgun, Phys. Rev. D 99, no. 8, 084012 (2019).

[77] W. Javed, A. Hazma and A. Övgün, Preprints 2019, 2019110142 (doi:10.20944/preprints201911.0142.v1)

[78] W. Javed, J. Abbas, and A. Övgün, Eur. Phys. J. C 79, no. 8, 694 (2019).

[79] A. Övgün, Phys. Rev. D 99, no. 10, 104075 (2019).

[80] W. Javed, j. Abbas and A. Övgün, Phys. Rev. D 100, no. 4, 044052 (2019).

[81] K. Jusufi and A. Övgün, Int. J. Geom. Meth. Mod. Phys. 16, no. 08, 1950116 (2019).

[82] G. Crisnejo, E. Gallo, and J. R. Villanueva, Phys. Rev. D 100, no. 4, 044006 (2019). 
[83] W. Javed, R. Babar, and A. Övgün, Phys. Rev. D 100, no. 10, 104032 (2019)

[84] K. de Leon and I. Vega, Phys. Rev. D 99, no. 12, 124007 (2019)

[85] Y. Kumaran and A. Övgün, arXiv:1905.11710 [gr-qc].

[86] W. Javed, J. Abbas, Y. Kumaran and A. Övgün, Preprints 2019, 2019110210 (doi: 10.20944/preprints201911.0210.v1).

[87] A. Övgün, I. Sakalli and J. Saavedra, arXiv:1908.04261 [gr-qc].

[88] Z. Li and A. Övgün, Preprints 2019, 2019110195 (doi: 10.20944/preprints201911.0195.v1)

[89] B. Ahmedov, B. Turimov, Z. Stuchlk and A. Tursunov, Int. J. Mod. Phys. Conf. Ser. 49, 1960018 (2019).

[90] B. Turimov, B. Ahmedov, A. Abdujabbarov and C. Bambi, arXiv:1802.03293 [gr-qc].

[91] A. Abdujabbarov, B. Ahmedov, N. Dadhich and F. Atamurotov, Phys. Rev. D 96, no. 8, 084017 (2017).

[92] J. Schee, Z. Stuchlk, B. Ahmedov, A. Abdujabbarov and B. Toshmatov, Int. J. Mod. Phys. D 26, no. 5, 1741011 (2017).

[93] M.J. Rees, Ann. Rev. Astron. Astrophys. 22, 471 (1984).

[94] J. Kormendy and D. Richstone, Ann. Rev. Astron. Astrophys. 33, 581 (1995).

[95] J.L. Synge, Mon. Not. R. Astron. Soc. 131, 463 (1966).

[96] J.P. Luminet Astron. Astrophys. 75, 228 (1979).

[97] J.M. Bardeen, in Black holes, in Proceeding of the Les Houches Summer School, Session 215239, edited by C. De Witt and B.S. De Witt and B.S. De Witt (Gordon and Breach, New York, 1973).

[98] S. Chandrasekhar, The Mathematical Theory of Black Holes (Oxford University Press, New York, 1992).

[99] H. Falcke, F. Melia and E. Agol, Astrophys. J. 528, L13 (2000).

[100] R. Kumar, S. G. Ghosh and A. Wang, arXiv:2001.00460 [gr-qc].

[101] R. A. Konoplya, arXiv:1912.10582 [gr-qc].

[102] R. Kumar, S. G. Ghosh and A. Wang, Phys. Rev. D 100, no. 12, 124024 (2019)

[103] R. A. Konoplya and A. Zhidenko, Phys. Rev. D 100, no. 4, 044015 (2019)

[104] A. Allahyari, M. Khodadi, S. Vagnozzi and D. F. Mota, arXiv:1912.08231 [gr-qc].

[105] R. A. Konoplya, Phys. Lett. B 795, 1 (2019)

[106] Z. Younsi, A. Zhidenko, L. Rezzolla, R. Konoplya and Y. Mizuno, Phys. Rev. D 94, no. 8, 084025 (2016)

[107] P. V. P. Cunha, N. A. Eir, C. A. R. Herdeiro and J. P. S. Lemos, arXiv:1912.08833 [gr-qc].

[108] Garattini, R. Eur. Phys. J. C (2019) 79: 951. https://doi.org/10.1140/epjc/s10052-019-7468-y

[109] B. Carter, Phys. Rev. 174, 1559 (1968).

[110] J.M. Bardeen, in Black holes, in Proceeding of the Les Houches Summer School, Session 215239, edited by C. De Witt and B.S. De Witt and B.S. De Witt (Gordon and Breach, New York, 1973).

[111] S. Chandrasekhar, The Mathematical Theory of Black Holes (Oxford University Press, New York, 1992). 